\title{
Upcoming Industrial Needs for Search
}

\author{
Gregory Grefenstette \\ EXALEAD \\ 10, place de la Madeleine \\ 75008 Paris \\ France \\ ggrefens@exalead.com
}

\begin{abstract}
Enterprise search and web searching have different goals and characteristics. Whereas internet browsing can sometimes be seen as a form of entertainment, enterprise search involves activities in which search is mainly a tool. People have work they want to get done. In this context, the idea of relevance in documents is different. Community can become as important as content in search. Work-related search engines of the future will provide much greater analysis and structuring of documents at index time, and searchers will have more powerful tools at retrieval time. We will discuss these and other trends, and show what new methods and techniques should be targeted to improve enterprise search.
\end{abstract}

